\title{
Tetanus Toxin Blocks the Exocytosis of Synaptic Vesicles Clustered at Synapses But Not of Synaptic Vesicles in Isolated Axons
}

\author{
Claudia Verderio, ${ }^{1}$ Silvia Coco, ${ }^{1}$ Alberto Bacci, ${ }^{1}$ Ornella Rossetto, ${ }^{2}$ Pietro De Camilli, ${ }^{3}$ Cesare Montecucco, ${ }^{2}$ \\ and Michela Matteoli ${ }^{1}$
}

${ }^{1}$ Consiglio Nazionale delle Ricerche Cellular and Molecular Pharmacology and B. Ceccarelli Centers, Department of Medical Pharmacology, 20129 Milano, Italy; 'Dipartimento di Scienze Biomediche, Consiglio Nazionale delle RicercheCentro Biomembrane, University of Padova, 35122 Padova, Italy, and ${ }^{3 H o w a r d ~ H u g h e s ~ M e d i c a l ~ I n s t i t u t e ~ a n d ~ D e p a r t m e n t ~}$ of Cell Biology, Yale University School of Medicine, New Haven, Connecticut 06510

Recycling synaptic vesicles are already present in isolated axons of developing neurons (Matteoli et al., 1992; Zakharenko et al., 1999). This vesicle recycling is distinct from the vesicular traffic implicated in axon outgrowth. Formation of synaptic contacts coincides with a clustering of synaptic vesicles at the contact site and with a downregulation of their basal rate of exo-endocytosis (Kraszewski et al., 1995; Coco et al., 1998) We report here that tetanus toxin-mediated cleavage of synaptobrevin/vesicle-associated membrane protein (VAMP2), previously shown not to affect axon outgrowth, also does not inhibit synaptic vesicle exocytosis in isolated axons, despite its potent blocking effect on their exocytosis at synapses. This differential effect of tetanus toxin could be seen even on different branches of a same neuron. In contrast, botulinum toxins $A$ and $\mathrm{E}$ [which cleave synaptosome-associated protein of $25 \mathrm{kDa}$.
(SNAP-25)] and $F$ (which cleaves synaptobrevin/VAMP1 and 2) blocked synaptic vesicle exocytosis both in isolated axons and at synapses, strongly suggesting that this process is dependent on "classical" synaptic SNAP receptor (SNARE) complexes both before and after synaptogenesis. A tetanus toxin-resistant form of synaptic vesicle recycling, which proceeds in the absence of external stimuli and is sensitive to botulinum toxin $F, E$, and $A$, persists at mature synapses. These data suggest the involvement of a tetanus toxin-resistant, but botulinum F-sensitive, isoform of synaptobrevin/VAMP in synaptic vesicle exocytosis before synapse formation and the partial persistence of this form of exocytosis at mature synaptic contacts.

Key words: exocytosis; synaptic vesicles; tetanus toxin; synaptogenesis; hippocampal neurons; synaptobrevin
Major characteristics of synaptic transmission are high spatial precision, speed, and great fidelity. These features are dependent on exocytosis taking place at restricted and well defined areas of the neuronal membrane. They rely on the presence of an extremely specialized machinery, allowing very rapid triggering and switching off of synaptic vesicle (SV) exocytosis in response to depolarization-evoked calcium influx (Barrett and Stevens, 1972). Regulated SV exocytosis at mature synaptic sites has been widely investigated, and several proteins that participate in this process have now been identified (Sollner et al., 1993; Bennett and Scheller, 1994; Ferro-Novick and Jahn, 1994; Südhof, 1995). It is well established that SV exocytosis involves the interaction of the synaptic vesicle membrane proteins synaptobrevin/vesicleassociated membrane protein (VAMP) 1 and 2 [v-SNAREs (soluble $N$-ethylmaleimide factor-attached protein (SNAP) receptors (SNARE)] with the plasma membrane proteins syntaxin and SNAP-25 (t-SNAREs). Synaptic SNARE proteins are targets for the proteolytic action of clostridial tetanus and botulinum neuro-

\footnotetext{
Received March 22, 1999; revised May 24, 1999; accepted May 24, 1999.

This work has been supported by Telethon Grants 1042 (to M.M.) and 1068 (to C.M.), by Human Frontier Science Program (to M.M. and P.D.C.), by the European Community Grants Biomed 2 BMH4 CT97 2410 (to C.M.) and BIO4-98-0408 (to M.M.), and by National Institutes of Health Grant NS36251 (to P.D.C.). We acknowledge Dr. R. Jahn (Gottingen, Germany) for gift of antibodies against synaptophysin, synaptobrevin/VAMP2, rab3a, and synaptotagmin.

Correspondence should be addressed to Michela Matteoli, Consiglio Nazionale delle Ricerche Cellular and Molecular Pharmacology, and B. Ceccarelli Centers, Department of Medical Pharmacology, University of Milano, via Vanvitelli 32, 20129 Milano, Italy.

Copyright (C) 1999 Society for Neuroscience $\quad 0270-6474 / 99 / 196723-10 \$ 05.00 / 0$
}

toxins (TeNT and BoNTs), which potently block exocytosis in nerve terminals (Blasi et al., 1993a,b; Schiavo et al., 1992, 1993a,b, 1995).

The molecular mechanisms involved in the development of CNS synapses are still poorly understood. A powerful experimental system to investigate these mechanisms is represented by primary cultures of hippocampal neurons. In these neurons, SVs, which release neurotransmitter and undergo high basal exoendocytotic recycling, are already present at very early developmental stages when axons grow in isolation. Formation of synaptic contacts coincides with a clustering of synaptic vesicles, with a downregulation of their basal recycling rate (Kraszewski et al., 1995; Coco et al., 1998), with a change in the calcium sensitivity of the exocytotic process (Coco et al., 1998), and with a switch in the population of calcium channels controlling neurotransmitter (glutamate) release (Scholz and Miller, 1995; Verderio et al., 1995).

Synaptic vesicle exocytosis, which occurs in developing axons, is clearly distinct from the constitutive exocytosis of vesicles that mediate axon elongation. Whereas the latter occurs primarily at the axon ending (Pfenninger and Maylie-Pfenninger 1981; Futerman et al., 1993; Craig et al., 1995; Zakharenko and Popov 1998), the former occurs along the entire distal axonal arbor (Matteoli et al., 1992; Kraszewski et al., 1995; Zakharenko et al., 1999). It was shown previously that axonal outgrowth and synaptogenesis in cultured CNS neurons is not inhibited by tetanus toxin (AhnertHilger et al., 1996; Osen Sand et al., 1996), strongly suggesting that the v-SNARE(s) implicated in this process are distinct from the classical synaptic v-SNARE(s). We report here that, surpris- 

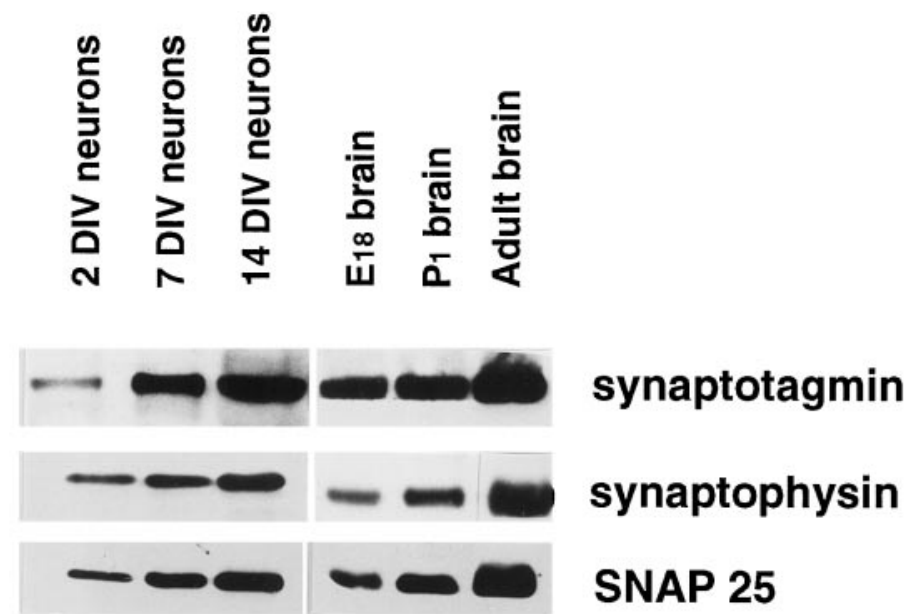

synaptophysin

SNAP 25

Figure 1. Synaptic protein expression during neuronal development. Western blot analysis of cell extracts from neuronal cultures maintained in vitro for 2,7 , and $15 \mathrm{~d}(D I V)$ and from rat brain at different developmental stages (E18, $\mathrm{P} 1$, and adult). SV (synaptophysin, synaptotagmin, synaptobrevin/VAMP2, and rab3a) and plasma membrane (SNAP-25 and syntaxin) proteins are expressed since early developmental stages and undergo a progressive increase in their expression in parallel with neuronal maturation. The same amount of material $(30 \mu \mathrm{g})$ has been loaded in each lane.

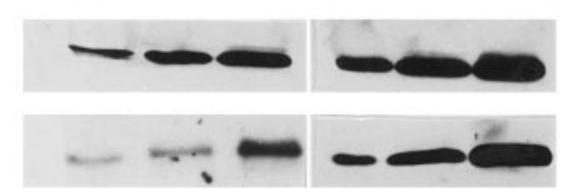

synaptobrevin/VAMP2 syntaxin

rab 3A ingly, even synaptic vesicle exocytosis is insensitive to the action of tetanus toxin in developing axons. This tetanus toxin-resistant form of SV recycling partially persists at mature synapses as spontaneous SV exocytosis. These findings suggest that the different properties of nonsynaptic and synaptic exocytosis of SVs correlate with a switch in v-SNARE isoforms underlying SNARE complex formation.

\section{MATERIALS AND METHODS}

Hippocampal cell cultures. Primary neuronal cultures were prepared from the hippocampi of 18-d-old fetal rats as described by Banker and Cowan (1977) and Bartlett and Banker (1984). Briefly, hippocampi were dissociated by treatment with trypsin $\left(0.25 \%\right.$ for $15 \mathrm{~min}$ at $\left.37^{\circ} \mathrm{C}\right)$, followed by trituration with a fire-polished Pasteur pipette. Dissociated cells were plated on poly-L-lysine-treated (Sigma, Milano, Italy) glass coverslips in MEM with $10 \%$ horse serum at densities ranging from 10,000 to 20,000 cells $/ \mathrm{cm}^{2}$. After few hours, coverslips were transferred to dishes containing a monolayer of cortical glial cells (Booher and Sensenbrenner, 1972), so that they were suspended over the glial cells but not in direct contact with them (Bartlett and Banker, 1984). Cells were maintained in MEM (Life Technologies, S. Giuliano, Italy) without sera, supplemented with $1 \% \mathrm{~N} 2$ (Life Technologies), $2 \mathrm{~mm}$ glutamine, and $1 \mathrm{mg} / \mathrm{ml}$ BSA (neuronal medium). A modification of the method used by Furshpan et al. (1976) was used to grow single neurons on small islands of substrate, consisting in a fine mist of poly-L-lysine sprayed on glass coverslips.

Experimental treatments. Neuronal cultures were exposed to $10 \mathrm{~nm}$ TeNT for $5 \mathrm{~min}$ in the presence or absence of $55 \mathrm{~mm} \mathrm{KCl}$ in the external medium, thoroughly washed, maintained in regular medium at $37^{\circ} \mathrm{C}$ for 2-18 hr, fixed, and double stained for synaptobrevin/VAMP2 and for synaptophysin. In some experiments, neurons were also exposed to $20 \mathrm{nM}$ BoNT/A, $80 \mathrm{~nm}$ BoNT/E, and 60-100 nm BoNT/F. An immunocytochemical assay based on the use of antibodies directed against the intravesicular domain of rat synaptotagmin I (Syt-ecto Abs) was used to test the efficacy of the toxins in blocking synaptic vesicle recycling. In particular, cultures were incubated with Syt-ecto Abs for $5 \mathrm{~min}$ or $1 \mathrm{hr}$ at $37^{\circ} \mathrm{C}$ in the presence or absence of $55 \mathrm{mM} \mathrm{KCl}$. Cells were then fixed with $4 \%$ paraformaldehyde in $0.12 \mathrm{M}$ phosphate buffer containing $0.12 \mathrm{M}$ sucrose for $25 \mathrm{~min}$ at $37^{\circ} \mathrm{C}$. Fixed cells were detergent-permeabilized and labeled with rhodamine-conjugated anti-rabbit antibodies as described previously (Matteoli et al., 1992). Counterstaining of neurons with antibodies directed against total synaptotagmin I (Syt mono), followed by fluorescein-conjugated anti-mouse antibodies, was performed as de- scribed previously (Matteoli et al., 1992). Coverslips were mounted in $70 \%$ glycerol in phosphate buffer containing $1 \mathrm{mg} / \mathrm{ml}$ phenylendiamine. Preparations were examined with a Zeiss (Oberkochen, Germany) microscope equipped with epifluorescence and photographed with T-MAX 400 (Kodak, Milano, Italy). Quantitative analysis was performed as described previously (Matteoli et al., 1996; Coco et al., 1998; Verderio et al., 1999a). For each experiment, 60-80 neurons were examined. Values obtained in different experiments were averaged and plotted.

Immunoblotting. Cultured hippocampal neurons were solubilized in $1 \%$ SDS, 5\% 2-mercaptoethanol, $65 \mathrm{~mm}$ Tris- $\mathrm{HCl}, \mathrm{pH} 6.8$, and $10 \%$ sucrose as described previously (Coco et al., 1997). SDS-PAGE electrophoresis and Western blotting were performed as described previously (Laemmli, 1970; Towbin et al., 1979). Briefly, cell extracts (30 $\mu \mathrm{g})$ were subjected to SDS-PAGE (10\% polyacrylamide gels) and transferred by electroblotting to nitrocellulose (Sartorius, Gottingen, Germany). Blots were blocked in $5 \%$ nonfat dry milk, $0.1 \%$ Tween $20,20 \mathrm{~mm}$ Tris, and $150 \mathrm{~mm} \mathrm{NaCl}, \mathrm{pH} 7.5$, at room temperature. Blots were incubated with antibodies for $2 \mathrm{hr}$ at room temperature in blocking buffer. Blots were then thoroughly washed, incubated $(1 \mathrm{hr})$ with horseradish peroxidaseconjugated anti-rabbit IgG or horseradish peroxidase-conjugated antimouse IgG (1:5000 in blocking buffer; Sigma), and finally washed with Tris-NaCl. The immunoreactive proteins were visualized with enhanced chemiluminescence (Amersham, Milano, Italy).

Antibodies. Rabbit polyclonal antibodies directed against the intravesicular domain of rat synaptotagmin I (Syt-ecto Abs) were generated as described previously (Matteoli et al., 1992) using a synthetic peptide corresponding to the residue 1-19 of the protein. Antibodies against synaptobrevin/VAMP1 and 2 were generated in rabbit as described previously (Rossetto et al., 1996). Polyclonal antibodies against synaptophysin and monoclonal antibodies against synaptobrevin/VAMP2, rab3a, and synaptotagmin I were a kind gift from Dr. R. Jahn (Gottingen, Germany). Polyclonal antibodies against syntaxin I and SNAP-25 were raised and used as described previously (Chilcote et al., 1995; Papini et al., 1995). Antirabbit rhodamine-conjugated antibodies were purchased from Boehringer Mannheim (Milano, Italy). Anti-mouse fluorescein-conjugated antibodies were from Jackson ImmunoResearch (West Grove, PA).

\section{RESULTS}

\section{Synaptic v- and t-SNAREs are already expressed by neurons at early developmental stages}

When maintained in primary cultures, embryonic hippocampal neurons develop through a series of well characterized develop- 
ctrl
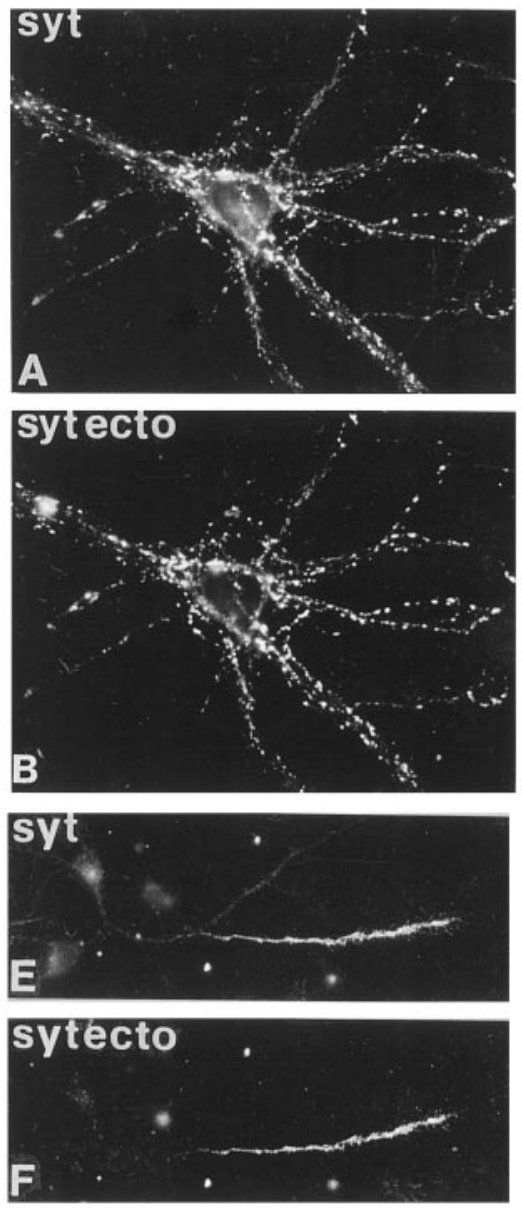

\section{TeNT}

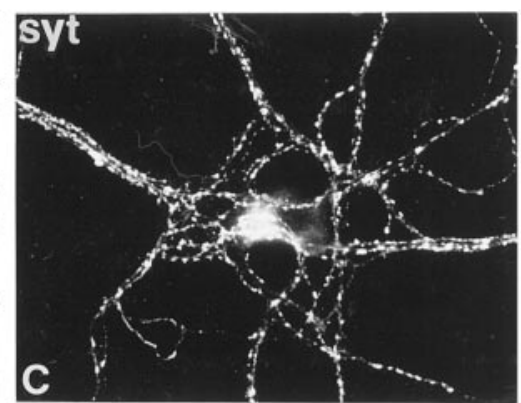

sytecto
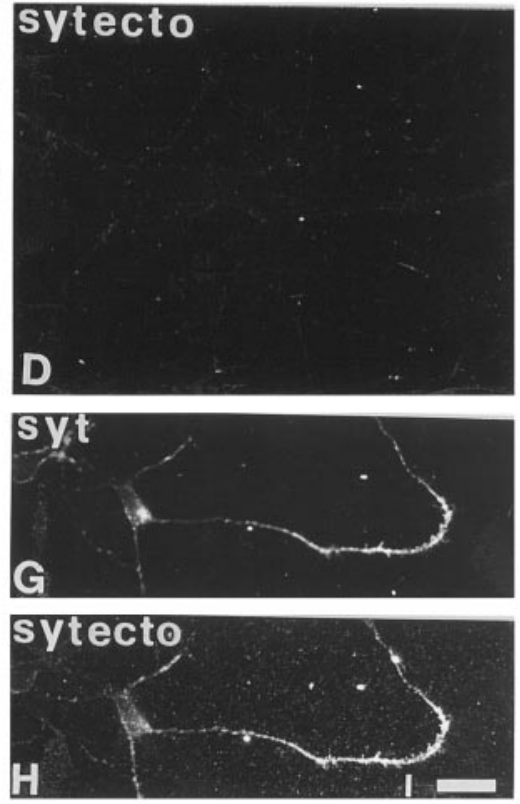

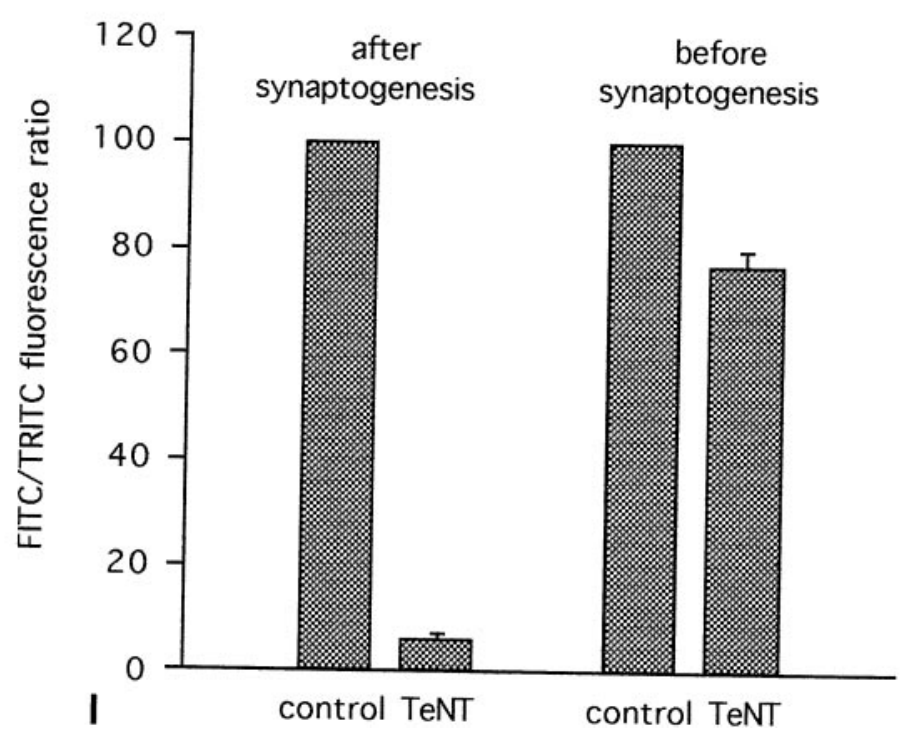

Figure 2. Tetanus toxin treatment inhibits SV exocytosis at mature synapses but not in developing neurons. $A, B$, Fifteenday-old neurons were incubated for $5 \mathrm{~min}$ in the presence of Syt-ecto Abs in $55 \mathrm{~mm}$ external $\mathrm{KCl}$ before $(A, B)$ or after $(C$, $D$ ) treatment with $10 \mathrm{~nm}$ TeNT. After this incubation, neurons were washed, fixed, detergent-permeabilized, reacted with rhodamine-conjugated goat anti-rabbit $\operatorname{IgGs}(B, D)$, and counterstained with antibodies against total synaptotagmin (syt), followed by FITC-conjugated goat anti-mouse $\operatorname{IgGs}(A$, $C)$. Puncta of immunoreactivity represent presynaptic nerve terminals, which outline perikarya and dendrites. Syt-ecto Abs are internalized at synaptic contacts when applied in control conditions $(B)$ but not after treatment with TeNT $(D) . E-H$, Exocytosis-dependent uptake of Syt-ecto Abs (applied for $5 \mathrm{~min}$ in the presence of $55 \mathrm{~mm} \mathrm{KCl}$ in the external medium) in living neurons before synaptogenesis, in control conditions $(F)$, or after treatment with $10 \mathrm{~nm}$ TeNT $(H) . E$, $G$, Double immunofluorescence of total synaptotagmin (syt) of the same neurons as in $F$ and $H$. Note that an efficient internalization of Syt-ecto Abs takes place in axons, even after treatment with TeNT $(H)$. Scale bar: $A-D, 20 \mu \mathrm{m} ; E-H$, $28 \mu \mathrm{m}$. I, Quantitative analysis of Syt-ecto internalization in neurons before and after synaptogenesis, both in control conditions or after treatment with $10 \mathrm{~nm}$ TeNT. mental stages (Dotti et al., 1988). After few days in culture, they establish a clear axo-dendritic polarity and, eventually, form a network of functional synaptic contacts characterized by presynaptic clusters of SVs and by the postsynaptic accumulation of glutamate receptors (Craig and Banker, 1994; Verderio et al., 1999b). Figure 1 shows that the SV proteins synaptotagmin I, synaptophysin, and rab3a, together with the three SNARE proteins synaptobrevin/VAMP2, SNAP-25, and syntaxin I, are already expressed by cultured hippocampal neurons at early developmental stages. For all of them, an increase in the levels of expression takes place in parallel with the time of differentiation in culture. A similar increase in synaptic protein expression was 
ctrl

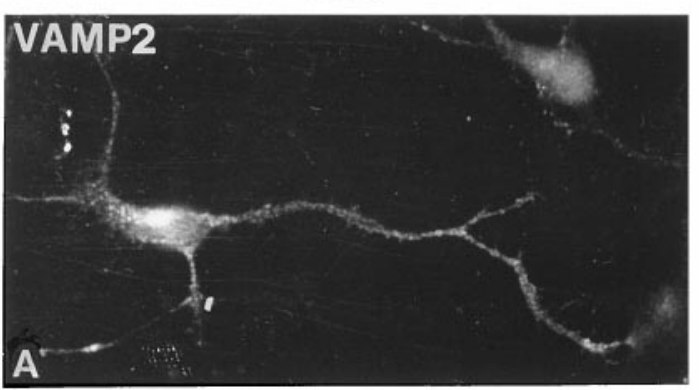

Figure 3. TeNT treatment cleaves synaptobrevin/VAMP2 without blocking SV recycling in neurons before synaptogenesis. A, B, Immature control neuron exposed to Syt-ecto Abs (for $5 \mathrm{~min}$ in the presence of $55 \mathrm{~mm} \mathrm{KCl}$ in the external medium) $(B)$ and double labeled for synaptobrevin/VAMP2 $(A)$. $C, D$, Immature neuron exposed to Syt-ecto Abs $(D)$ and double labeled for synaptobrevin/ VAMP2 $(C)$ after treatment with $10 \mathrm{~nm}$ TeNT. Note that TeNT treatment cleaves synaptobrevin/VAMP2 without impairing SV recycling. Scale bar, $11.25 \mu \mathrm{m}$. $E$, Quantitative analysis of Syt-ecto Ab internalization (-) and synaptobrevin/ VAMP2 cleavage ( $\square$ ) in developing neurons at different times after culture intoxication. Note that TeNT treatment dramatically reduces synaptobrevin/ VAMP2 immunoreactivity over time without significantly reducing SV recycling. $F$, Quantitative analysis of Syt-ecto $\mathrm{Ab}$ internalization (-) and synaptobrevin/VAMP2 cleavage $(\square)$ in developing neurons exposed to increasing doses of BoNT/F. Note the existence of a strict correlation between synaptobrevin/ VAMP2 cleavage and inhibition of SV recycling. Values were expressed as a ratio between the signals produced by Sytecto Abs or by synaptobrevin/VAMP2 antibodies and those produced by antibodies directed against total synaptotagmin I or synaptophysin.
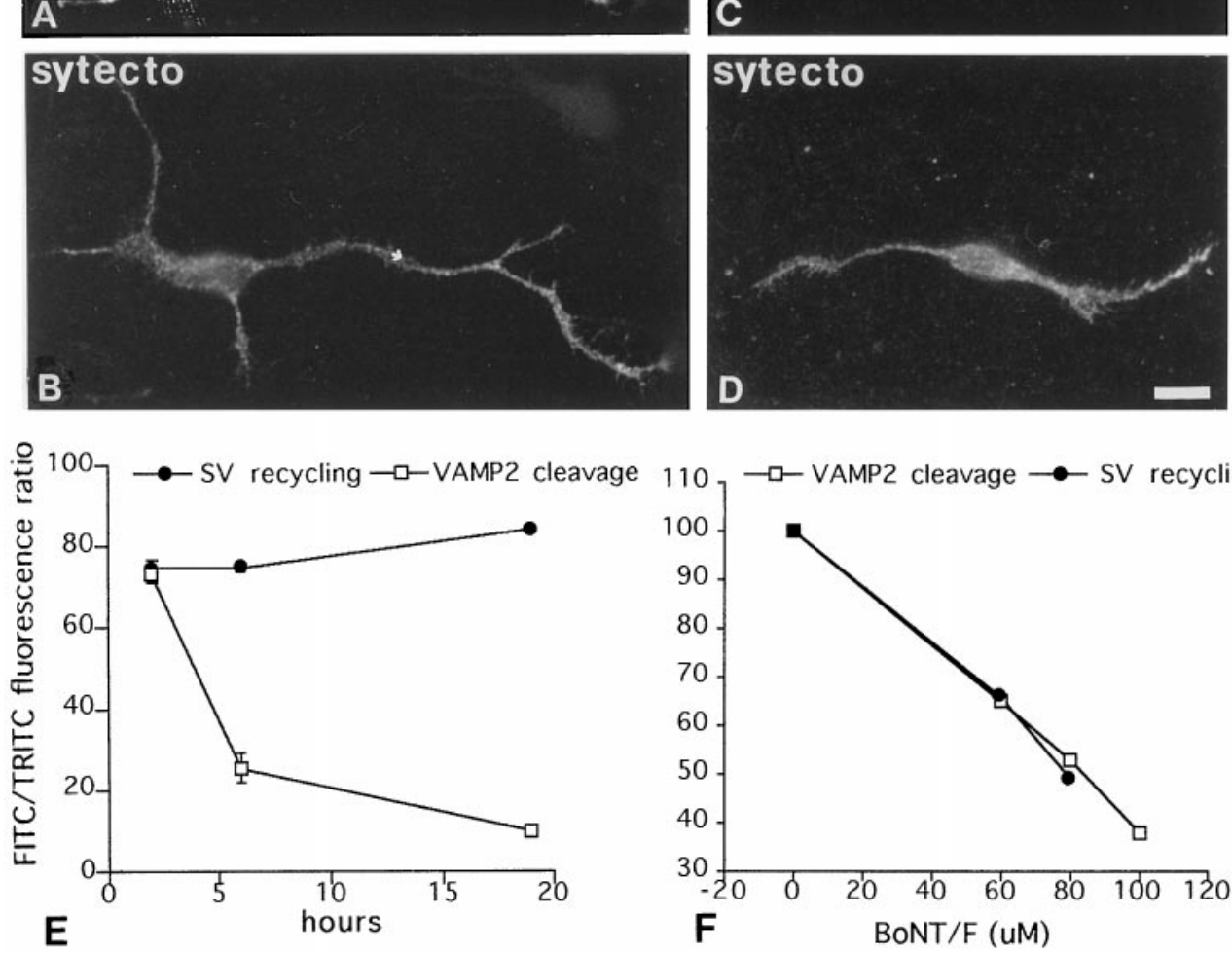

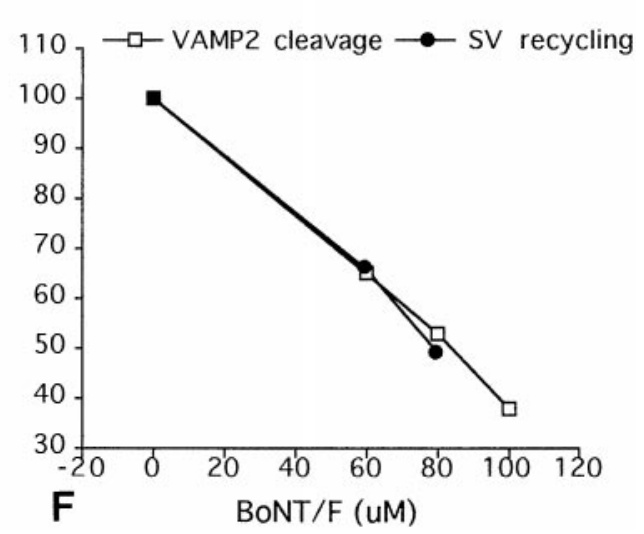

found to take place also during brain development, as indicated by Western blot analysis of total homogenates from embryonic day 18 (E18), postnatal day (P1), and adult rat brains. In contrast, we could not detect any synaptobrevin/VAMP1 immunoreactivity in cultured hippocampal neurons before synaptogenesis (data not shown). Immunofluorescence experiments revealed that, before synaptogenesis, synaptobrevin/VAMP2 immunoreactivity was localized on vesicular structures dispersed along the axon (see Fig. $3 A$ ). These vesicular structures, which are immunoreactive for synaptotagmin I (see Fig. 3B), synaptophysin, and rab3a (data not shown), have been already identified as bona fide SVs (Fletcher et al., 1991; Matteoli et al., 1991, 1992, 1996; Kraszewski et al., 1995; Coco et al., 1998). Syntaxin I and SNAP-25 were found to be present throughout all neuronal compartments (data not shown) (Galli et al., 1995).

\section{TeNT blocks recycling of SVs clustered at synapses but does not block SV recycling before synaptogenesis}

To monitor SV recycling, we used an assay based on antibodies directed against the intravesicular domain of the SV protein synaptotagmin I (Syt-ecto Abs), which become internalized in the lumen of SVs when they undergo exocytosis and compensatory endocytosis (Matteoli et al., 1992). As already shown, Syt-ecto Abs become internalized by an activity-dependent mechanism at synaptic contacts of cultured hippocampal neurons (Fig. 2B) and are also actively taken up by recycling vesicles in the axon of developing neurons (Fig. $2 F$ ). Mature cultures were incubated with $10 \mathrm{nM}$ TeNT for 5 min under depolarizing conditions and assayed for SV recycling $2 \mathrm{hr}$ later, when the TeNT substrate synaptobrevin/VAMP2 is completely cleaved (Matteoli et al., 1996). Virtually no internalization of Syt-ecto Abs at synaptic sites was detected ( $n=15$ experiments) (Fig. $2 D, I$, quantitative analysis), consistent with a block of exocytosis produced by TeNT treatment. In contrast, when applied to neurons before synaptogenesis, TeNT was actively internalized (data not shown) (Matteoli et al., 1996) but did not produce any relevant inhibitory effect on SV recycling $(n=7)$ (Fig. $2 H, I$, quantitative analysis).

The lack of effect of TeNT on SV recycling in neurons before synaptogenesis could result from inaccessibility of synaptobrevin/ VAMP2 to the proteolytic action of the toxin. To test this possibility, hippocampal neurons were stained for synaptobrevin/ VAMP2 after TeNT treatment. In control neurons, synaptobrevin/VAMP2 was localized on vesicles dispersed throughout the distal axonal arbor (Fig. 3A). These vesicles colocalized with internalized Syt-ecto Abs (Fig. 3B), thus supporting their identification as recycling SVs. After $6 \mathrm{hr}$ of TeNT treatment, a substantial reduction (up to $77 \%$ ) of synaptobrevin/VAMP 2 stain- 

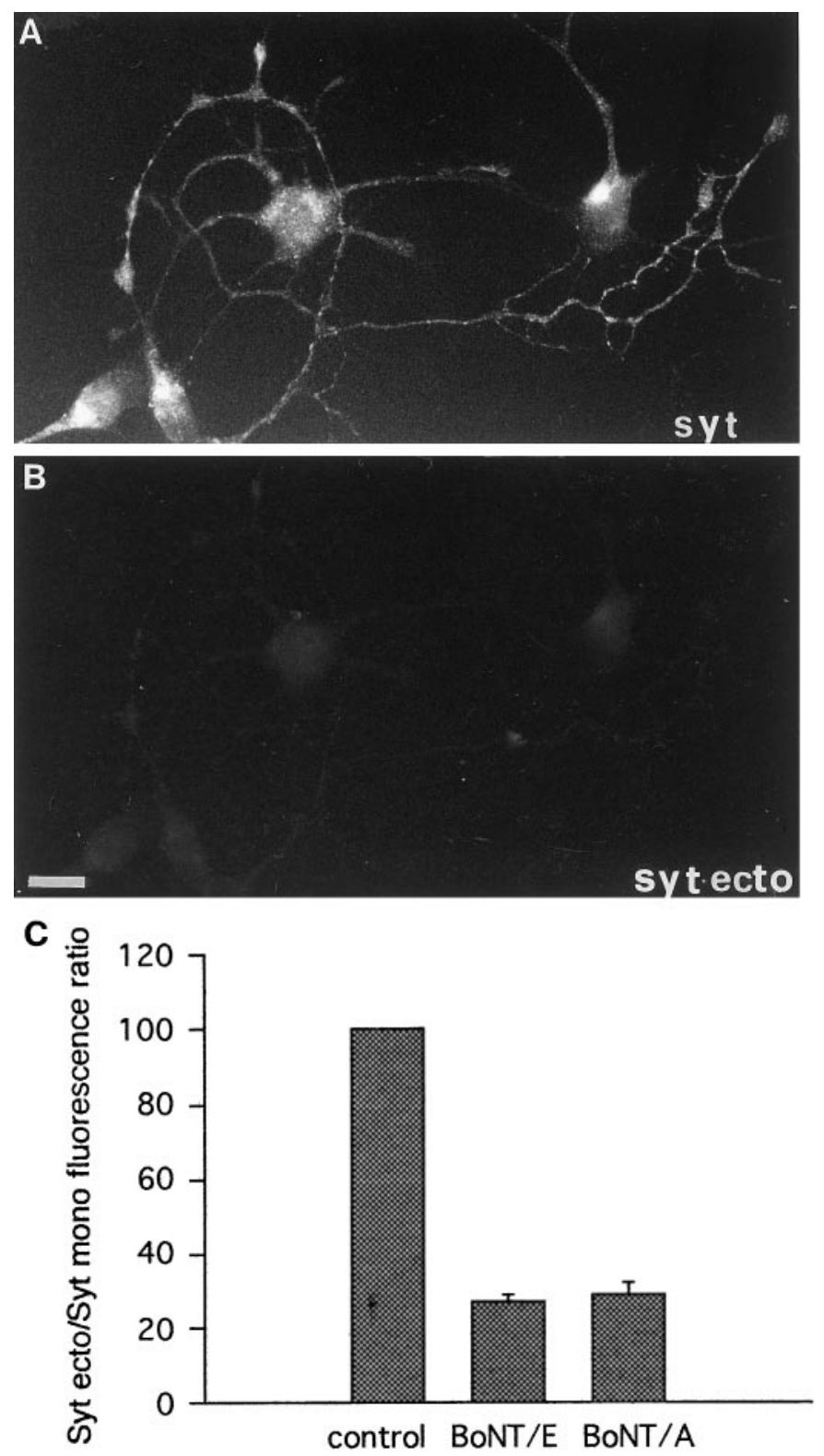

Figure 4. BoNT/A and E block SV recycling in neurons before synaptogenesis. $A, B$, Exocytosis-dependent uptake of Syt-ecto Abs (applied for $5 \mathrm{~min}$ in the presence of $55 \mathrm{~mm} \mathrm{KCl}$ in the external medium) in immature neurons after treatment with $20 \mathrm{~nm}$ BoNT/A. Virtually no internalization takes place in neuronal processes $(B)$, visualized by double labeling with antibodies against total synaptotagmin $(s y t, A)$. Scale bar, $12.8 \mu \mathrm{m}$. $C$, Quantitative analysis of Syt-ecto internalization in cultures treated with $20 \mathrm{~nm} \mathrm{BoNT/A}$ and $80 \mathrm{~nm}$ BoNT/E. Note that BoNTs of both serotypes strongly reduce SV recycling.

ing was detected (Fig. 3C, E, quantitative analysis). An even more pronounced cleavage (up to $90 \%$ ) was obtained $18 \mathrm{hr}$ after TeNT treatment (Fig. 3E). Surprisingly, however, this reduction did not correlate with an impairment in SV recycling (Fig. $3 E$, quantitative analysis). In contrast, BoNT/F, which cleaves VAMP2 as well but at a different peptide bond, significantly inhibited SV exocytosis in developing axons in a dose-dependent manner (Fig. $3 F$ ). Moreover, when hippocampal cultures at early developmental stages were incubated in the presence of $20 \mathrm{~nm} \mathrm{BoNT/A}$ or $80 \mathrm{nM}$ BoNT/E, which both cleave SNAP-25 (Blasi et al., 1993a; Schiavo et al., 1993a), a substantial reduction of Syt-ecto Ab internaliza- tion could be detected ( $n=6$ ) (Fig. $4 B, C$, quantitative analysis). Thus, SV exocytosis before synaptogenesis requires SNAP-25 and a TeNT-insensitive, but BoNT/F-sensitive, isoform of synaptobrevin/VAMP2 isoform.

\section{TeNT insensitivity is not related to the maturation stage of neurons in culture}

Hippocampal neurons maintained in vitro for 2-3 weeks are endowed with a dense network of synaptic contacts. However, a few isolated axons containing dispersed SVs and lacking a postsynaptic target are occasionally detectable in mature cultures. Figure 5, $A$ and inset a, show examples of these isolated axons in cultures of synaptically connected neurons that have been labeled with antibodies directed against total synaptotagmin (Fig. $5 A$ ) or with antibodies directed against synaptophysin (inset $a$ ). SVs present in these isolated axons of otherwise mature neurons undergo an active exo-endocytotic recycling, as demonstrated by the active internalization of the Syt-ecto Abs (Fig. 5B). Treatment of mature cultures with TeNT produced an efficient cleavage of synaptobrevin/VAMP2 in the isolated axons (inset $b$ ), similar to what has been already shown for synaptobrevin/VAMP2 present at synaptic contacts (Matteoli et al., 1996). However, whereas TeNT strongly inhibited SV fusion at sites of synaptic contacts (Figs. 2D, I; 5B, arrowheads), it did not substantially reduce vesicle recycling in isolated axons (Fig. $5 B$ ). This observation argues against the possibility that the lack of TeNT effect on SV recycling before synaptogenesis is related to the immature stage of the neurons.

To further prove this point, we investigated whether different axonal branches of the same neuron may exhibit different sensitivity to the toxin. To this aim, hippocampal neurons were cultured on poly-L-lysine microislands, i.e., under conditions that allow the analysis of single neurons whose axons form autaptic contacts (Furshpan et al., 1976; Bekkers and Stevens, 1991). These single neurons often extend additional axons that lack a postsynaptic target, thus allowing a simultaneous analysis of synaptic and nonsynaptic SV exocytosis in a same cell (Fig. $5 \mathrm{C}-H$ ). We found that exposure of these neurons to TeNT produced the typical inhibition of SV recycling at sites of synaptic contacts (Fig. $5 D$, small arrowheads, $F$ ). On the other hand, SV recycling in isolated processes was not impaired (Fig. 5D, large arrowhead, H).

\section{A TeNT-insensitive form of SV recycling persists at mature synapses}

Mature cultures were incubated with TeNT and assayed for evoked SV recycling $2 \mathrm{hr}$ later. A residual internalization of Syt-ecto Abs was detectable in $<10 \%$ of synaptic sites $(n=15)$ (Fig. 6C, evoked), consistent with a massive cleavage of the TeNT substrate synaptobrevin/VAMP2 (Matteoli et al., 1996). On the other hand, after TeNT intoxication, the majority of synapses (82\%) maintained a residual SV recycling, resulting in a significant level of Syt-ecto Ab internalization after $1 \mathrm{hr}$ incubation in the absence of depolarizing stimuli and in the presence of TTX $(n=4)$ (Fig. 6B, C, spontaneous). In contrast, pretreatment of mature cultures with BoNT/F, BoNT/E, or BoNT/A impaired to a similar extent Syt-ecto Ab internalization attributable to either spontaneous or depolarization-evoked SV recycling (Fig. 6C).

\section{DISCUSSION}

Bona fide SVs are present in isolated processes of developing neurons before synapse formation. These vesicles have the same size range of typical presynaptic SVs (Matteoli et al., 1992; Kraszewski et al., 1995), store and secrete neurotransmitter 

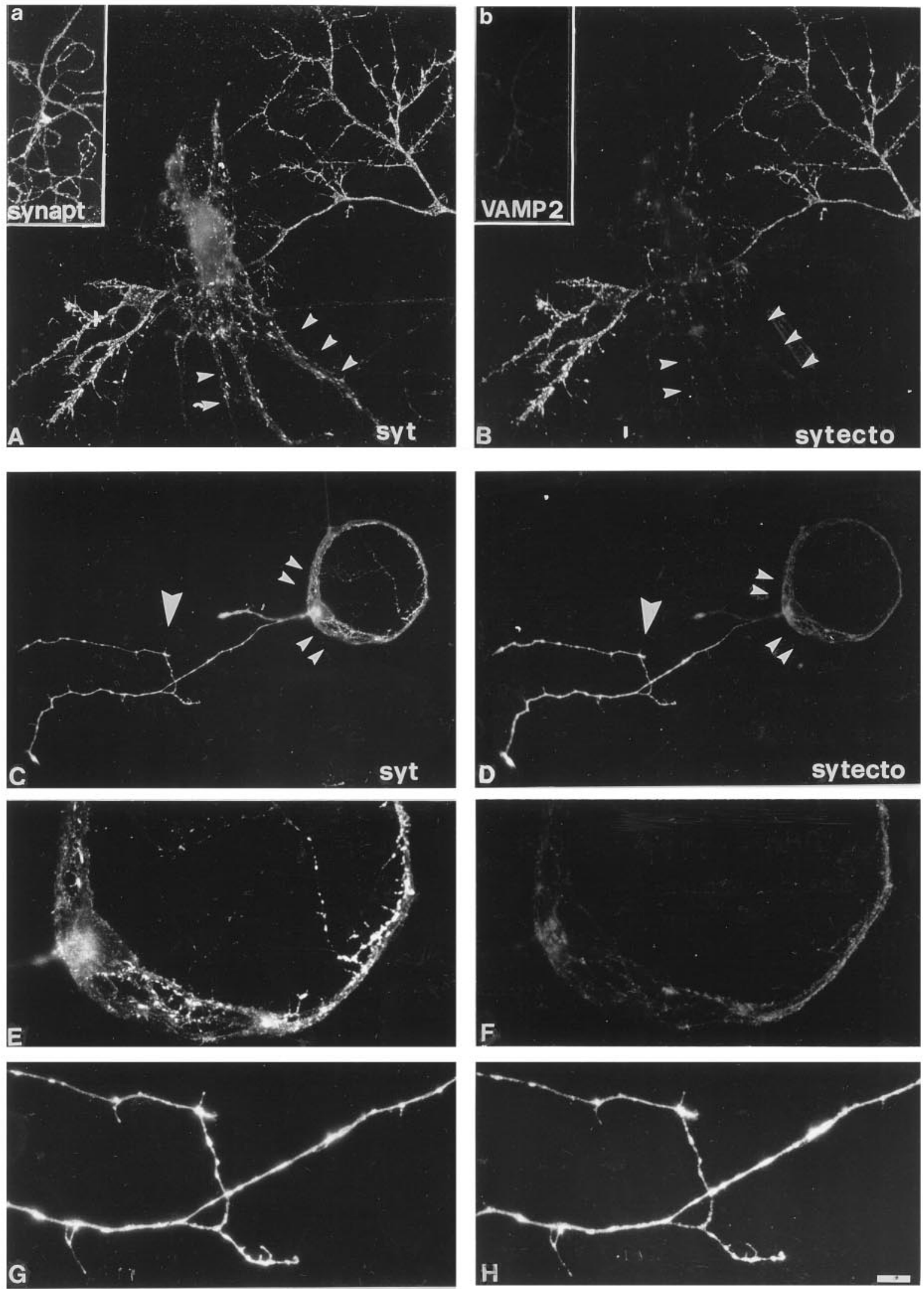

Figure 5. SV recycling is TeNT-insensitive in isolated axons of mature cultures. An efficient internalization of Syt-ecto Abs, applied for 5 min in 55 mM $\mathrm{KCl}$, takes place in the isolated axons present in fully differentiated cultures after exposure to $10 \mathrm{~nm}$ TeNT $(B)$. No labeling is detectable at synaptic contacts (arrowheads). A, Double labeling of the same culture as in $B$ with antibodies against total synaptotagmin (syt). Insets $a, b$, After TeNT intoxication, synaptobrevin/VAMP2 immunoreactivity is no longer visible in an isolated axon (b) double labeled with the (Figure legend continues) 
(Young and Poo 1983; Sun and Poo, 1987; Zakharenko et al., 1999), and, based on immunocytochemical results, have an SV protein composition (Fletcher et al., 1991; Matteoli et al., 1991, 1992). In developing processes, these vesicles are organized in small clusters that, similar to SVs of the presynapse, are disrupted by okadaic acid (Kraszewski et al., 1995). Moreover, they are excluded from the leading edge of the growth cone (Kraszewski et al., 1995), indicating that they do not participate to the elongation of the axon. Finally, they undergo active exo-endocytotic recycling (Matteoli et al., 1992; Kraszewski et al., 1995; Coco et al., 1998), and their fusion with the plasma membrane is partially stimulated by depolarization (Kraszewski et al., 1995; Coco et al., 1998; Zakharenko et al., 1999).

We used a morphological assay (Matteoli et al., 1992) to follow SV dynamics after clostridial toxin treatment, independent from possible effects of these toxins on postsynaptic exocytosis and responsiveness (Lledo et al., 1998, Maletic-Savatic et al., 1998; Maletic-Savatic and Malinow, 1998; Nishimune et al., 1998; Osten et al., 1998; Song et al., 1998). Our results demonstrate that, different from SV exocytosis at mature synapses, SV recycling before synaptogenesis is not significantly inhibited by treatment with TeNT. The lack of TeNT effect on SV recycling before synaptogenesis is not because of a poor penetration of the toxin in immature neurons, because an efficient internalization of TeNT has been shown to occur in neurons already before synaptogenesis (Matteoli et al., 1996). On the other hand, the resistance of SV recycling to treatment with TeNT does not result from inaccessibility of the substrate synaptobrevin/VAMP2 to the toxin itself. Indeed, a substantial cleavage of synaptobrevin/ VAMP2 is achieved by TeNT treatment in neurons before synaptogenesis, although a more prolonged exposure to the toxin with respect to neurons after formation of synaptic contacts is required. Because TeNT enters SVs before synaptogenesis with a high rate (Matteoli et al., 1996), the reduced efficacy of TeNT in cleaving synaptobrevin/VAMP2 in immature neurons may be attributable to a less efficient translocation of the toxin in the cytosol and/or to the higher turnover of the protein in neurons at early developmental stages (Daly and Ziff, 1997).

The persistence of SV exo-endocytotic recycling in hippocampal neurons before synaptogenesis after TeNT treatment raises at least three possibilities. The first is that SV recycling is supported by a fraction of synaptobrevin/VAMP2, which, being shielded by a tight complex with other proteins because of post-translational modifications, cannot be cleaved by the toxin. However, because the percentage of cleaved synaptobrevin/VAMP2 and the inhibition of SV recycling are unrelated, this possibility is unlikely. The second is that SV fusion may proceed in the absence of synaptobrevin/VAMP2. It has been shown in yeast that organelles endowed with syntaxin homologs, but not synaptobrevin/VAMP2 homologs, may fuse each other (Nichols et al., 1997). Although it has been demonstrated that pools of syntaxin I and SNAP-25 may be present on SV membranes (WalchSolimena et al., 1995), the relatively low rate of fusion in the absence of synaptobrevin/VAMP2 (Nichols et al., 1997) does not fit with the high efficiency of SV recycling before synaptogenesis
(Kraszewski et al., 1995; Coco et al., 1998). The third is that a TeNT-resistant isoform of synaptobrevin/VAMP2 may mediate $\mathrm{SV}$ exocytosis at early developmental stages. The finding that BoNT/F-induced cleavage of synaptobrevin/VAMP2 correlates with inhibition of SV recycling strongly supports this third hypothesis, suggesting the existence of a synaptobrevin/VAMP isoform, specifically lacking the cleavage or recognition site for TeNT and mostly supporting exocytosis before synaptogenesis. The involvement of the SNARE fusion complex proteins in SV exocytosis before synaptogenesis is further supported by the finding that SV recycling, although being not reduced by TeNT, is strongly inhibited by BoNT/A and BoNT/E, which cleave SNAP-25, the other protein component that, together with synaptobrevin/VAMP2 and syntaxin, mediates fusion of SVs at mature synapses.

When synapses form, SVs enter a regulated, synaptobrevin/ VAMP2-dependent exocytotic pathway. However, the acquisition of a TeNT-sensitive exocytosis does not correlate with a complete elimination of the TeNT-insensitive mechanism of SV recycling. Indeed, a residual TeNT-resistent and action potentialindependent SV recycling is maintained in the majority $(85 \%)$ of the synapses. The existence of a TeNT-insensitive form of exocytosis at mature synapses has been already reported (Duchen and Tonge 1973; Dreyer and Schmitt 1981; Dreyer 1989; Herreros et al., 1995; Sweeney et al., 1995; Capogna et al., 1997). In some experimental models, TeNT-resistant exocytosis appears to entirely account for the spontaneous neurotransmitter release (Bevan and Wendon, 1984; Hua et al., 1998). In other systems, application of TeNT produces a relevant decrease but never a complete block of spontaneous events (with a single exception reported by Mellanby and Thompson, 1972). In our experimental model, although strongly reduced, miniature EPSC frequency was not completely blocked, whereas BoNT/E completely inhibited miniature spontaneous activity (our unpublished observations). This observation is in agreement with the results obtained with the Syt-ecto Ab presynaptic assay, which demonstrate that the majority of synaptic contacts that are able to recycle SVs after TeNT treatment do not recycle SVs after treatment with BoNT/A, E, and, F, suggesting the involvement of the same machinery operating before synaptogenesis. It is noteworthy that, in Drosophila synaptobrevin mutants, the evoked release is entirely abolished, whereas only a fraction of spontaneous release is inhibited (Deitcher et al., 1998). On the other hand, both evoked and spontaneous release are disrupted by cleavage of syntaxin I (Schulze et al., 1995).

The mechanism responsible for altering the balance between the TeNT-insensitive and the TeNT-sensitive pathway during synaptogenesis is not clear yet. It is possible to hypothesize that the presence of a postsynaptic target plays a role in favoring exocytosis being predominantly controlled by high rises in the levels of calcium (Coco et al., 1998) and mostly relying on synaptobrevin/VAMP2. This possibility is supported by results obtained in single neurons growing in microislands. In this experimental model, the same neuron may be endowed with two distinct SV-containing compartments: the typical presynaptic terminals

\section{$\longleftarrow$}

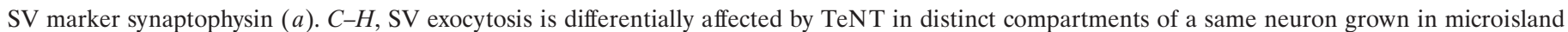

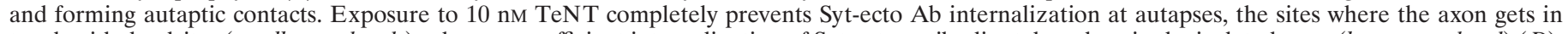

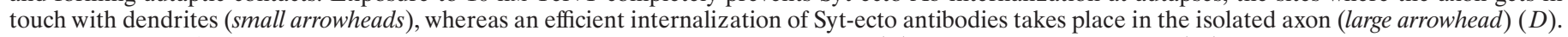

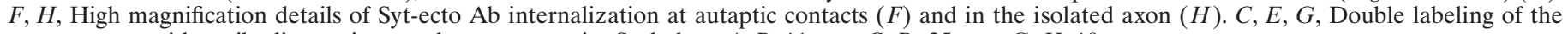
same neuron with antibodies against total synaptotagmin. Scale bar: $A, B, 11 \mu \mathrm{m} ; C, D, 25 \mu \mathrm{m} ; G, H, 10 \mu \mathrm{m}$. 

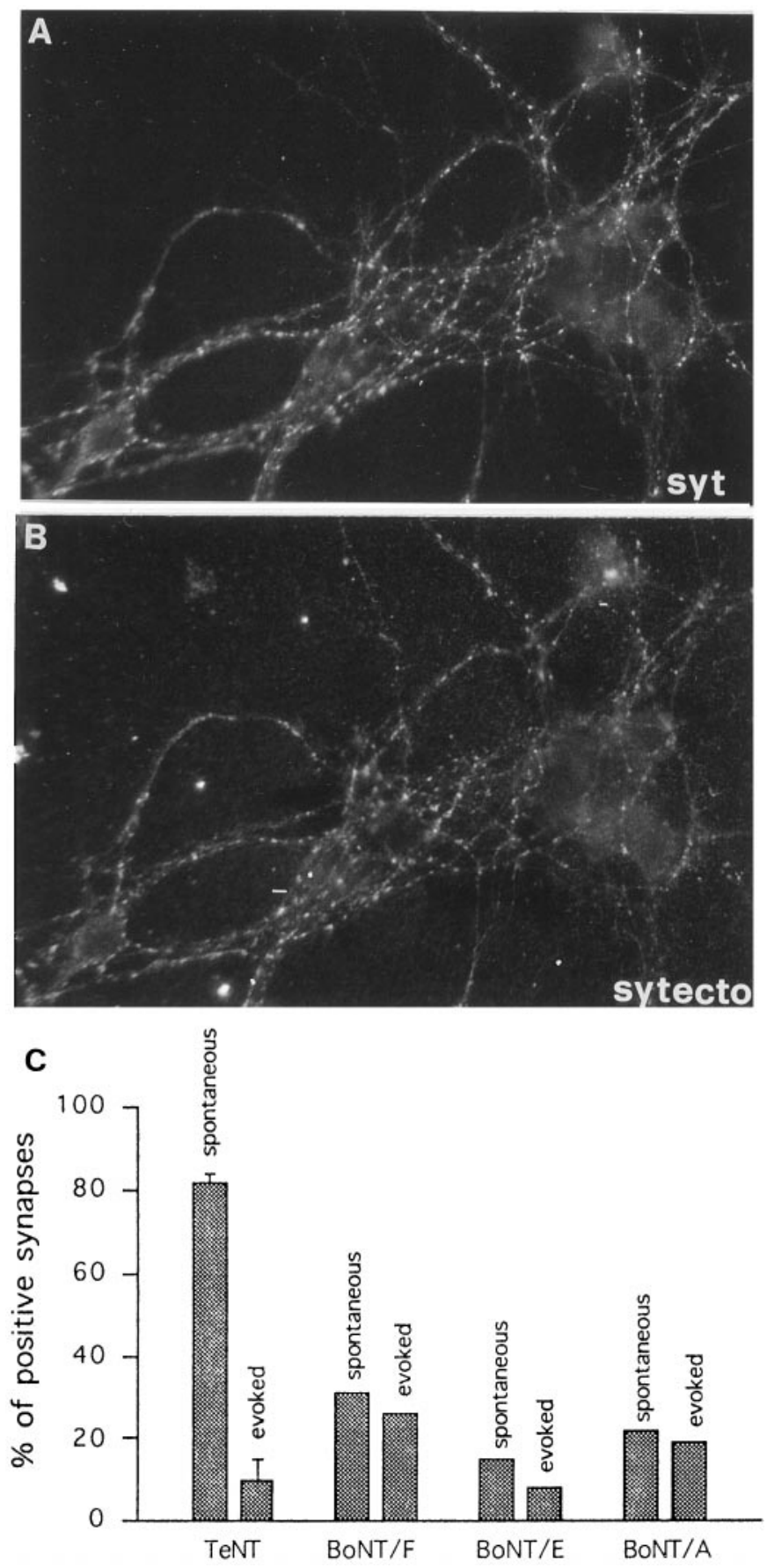

Figure 6. Persistence of spontaneous release at the synapse after TeNT but not BoNT treatment. $A, B$, Spontaneous Syt-ecto internalization at synaptic sites in TeNT-poisoned neurons $(B)$ double labeled with antibodies against total synaptotagmin $(s y t, A)$. C, Quantitative analysis of Syt-ecto $\mathrm{Ab}$ internalization in mature neurons intoxicated with TeNT or with BoNTs (20 nm BoNT/A, $80 \mathrm{~nm}$ BoNT/E, and $80 \mathrm{~nm}$ BoNT/F). Note that BoNTs of all serotypes prevent similar spontaneous and evoked Syt-ecto $\mathrm{Ab}$ internalization at the majority of synaptic contacts, whereas TeNT inhibits evoked Syt-ecto internalization without substantially impairing spontaneous uptake. Incubation with Syt-ecto antibodies is performed for $5 \mathrm{~min}$ in the presence of $55 \mathrm{mM} \mathrm{KCl}$ in the external medium or for $1 \mathrm{hr}$ in low $\mathrm{KCl}(5 \mathrm{~mm})$ and $1 \mu \mathrm{M}$ TTX. containing clustered vesicles, and the isolated axon characterized by diffusely distributed SVs. Strikingly, whereas SV recycling is sensitive to TeNT at sites of synaptic contacts, it is not inhibited in the isolated axon of the same cell, indicating that the same neuron may differently regulate exocytosis occurring in two different compartments, possibly in relation with the presence of a postsynaptic target. Several data, recently obtained in different experimental systems, suggest that indeed maturation of presynaptic structure and function is affected by signals from the postsynaptic cell (Fletcher et al., 1994; Haydon and Zoran, 1994; Campagna et al., 1995; Dechiara et al., 1996; Petersen et al., 1997; Davis et al., 1998; Fitzsimonds and Poo, 1998). Our data support the possibility that the postsynaptic cell may actively affect the presynaptic machinery controlling SV fusion during synaptogenesis between neurons in the mammalian CNS.

\section{REFERENCES}

Ahnert-Hilger G, Kutay U, Chahoud I, Rapoport T, Wiedenmann B (1996) Synaptobrevin is essential for secretion but not for the development of synaptic processes. Eur J Cell Biol 70:1-11.

Banker GA, Cowan WM (1977) Rat hippocampal neurons in dispersed cell culture. Brain Res 126:379-425.

Barrett EF, Stevens CF (1972) The kinetics of transmitter release at the frog neuromuscular junction. J Physiol (Lond) 227:691-708.

Bartlett W P, Banker GA (1984) An electron microscopic study of the development of axon and dendrites by hippocampal neurons in culture. II. Synaptic relationships. J Neurosci 4:1954-1965.

Bekkers JM, Stevens CF (1991) Excitatory and inhibitory autaptic currents in isolated hippocampal neurons maintained in cell cultures. Proc Natl Acad Sci USA 88:7834-7838.

Bennet MK, Scheller RH (1994) Molecular correlates of synaptic vesicles docking and fusion. Curr Opin Neurobiol 4:324-329.

Bevan S, Wendon LMB (1984) A study of the action of tetanus toxin at rat soleous neuromuscular junction. J Physiol (Lond) 348:1-17.

Blasi J, Chapman ER, Yamasaki S, Binz T, Niemann H, Jahn R (1993a) Botulinum neurotoxin $\mathrm{C} 1$ blocks neurotransmitter release by means of cleaving HPC-1/syntaxin. EMBO J 12:4821-4828.

Blasi J, Chapman ER, Link E, Binz T, Yamasaki S, De Camilli P, Sudhof TC, Niemann H, Jahn R (1993b) Botulinum neurotoxin A selectively cleaves the synaptic protein SNAP-25. Nature 365:160-163.

Booher J, Sensenbrenner M (1972) Growth and cultivation of dissociated neurons and glial cells from embryonic chick, rat, and human brain in flask cultures. Neurobiology 2:97-105.

Campagna JA, Ruegg MA, Bixby JL (1995) Agrin is a differentiation-inducing "stop signal" for motoneurons in vitro. Neuron 15:1365-1374.

Capogna M, McKinney RA, O'Connor V, Gahwiler BH, Thompson $\mathrm{SM}$ (1997) $\mathrm{Ca}^{2+}$ or $\mathrm{Sr}^{2+}$ partially rescues synaptic transmission in hippocampal cultures treated with botulinum toxin $\mathrm{A}$ and $\mathrm{C}$, but not tetanus toxin. J Neurosci 17:7190-7202.

Chilcote TJ, Galli T, Mundigl O, Edelmann L, McPherson PS, Takei K, De Camilli P (1995) Cellubrevin and synaptobrevins: similar subcellular localization and biochemical properties in PC12 cells. J Cell Biol 129:219-231.

Coco S, Verderio C, Trotti D, Rothstein JD, Volterra A, Matteoli M (1997) Non-synaptic localization of the glutamate transporter EAAC1 in cultured hippocampal neurons. Eur J Neurosci 9:1902-1910.

Coco S, Verderio C, De Camilli P, Matteoli M (1998) Calcium dependence of synaptic vesicle recycling before and after synaptogenesis. J Neurochem 71:1987-1992.

Craig AM, Banker G (1994) Neuronal polarity. Annu Rev Neurosci $17: 267-310$.

Craig AM, Wyborski RJ, Banker J (1995) Preferential addition of newly synthesized membrane protein at axonal growth cones. Nature 375:592-594.

Daly C, Ziff EB (1997) Post-trascriptional regulation of synaptic vesicle protein expression and the developmental control of synaptic vesicle formation. J Neurosci 17:2365-2375.

Davis GW, DiAntonio A, Petersen SA, Goodman CS (1998) Postsynaptic PKA controls quantal size and reveals a retrograde signal that 
regulates presynaptic transmitter release in Drosophila. Neuron 20:305-315.

Dechiara TM, Bowen DC, Valenzuela DM, Simmons MV, Poueymirou WT, Thomas S, Kinetz E, Compton DL, Rojas E, Park JS (1996) The receptor tyrosine kinase MuSK is required for neuromuscular formation in vivo. Cell 85:501-512.

Deitcher DL, Ueda A, Stewart BA, Burgess RW, Kikodoro Y, Schwartz TL (1998) Distinct requirements for evoked and spontaneous release of neurotransmitter are revealed by mutations in the Drosophila gene neuronal-synaptobrevin. J Neurosci 18:2028-2039.

Dotti CG, Sullivan CA, Banker GA (1988) The establishment of polarity by hippocampal neurons in culture. J Neurosci 8:1454-1468.

Dreyer F (1989) Peripheral actions of tetanus toxin. In: Botulinum neurotoxin and tetanus neurotoxin (Simpson LL, ed), pp 179-202. San Diego: Academic.

Dreyer F, Schmitt A (1981) Different effects of botulinum A toxin and tetanus toxin on the transmitter releasing process at the mammalian neuromuscular junction. Neurosci Lett 26:307-311.

Duchen LW, Tonge DA (1973) The effects of tetanus toxin on neuromuscular transmission and on the morphology of motor endplates in slow and fast skeletal muscle of the mouse. J Physiol (Lond) 228:157-172.

Ferro-Novick S, Jahn R (1994) Vesicle fusion from yeast to man. Nature 370:191-193.

Fitzsimonds RM, Poo MM (1998) Retrograde signaling in the development and modification of synapses. Physiol Rev 78:143-170.

Fletcher TL, Cameron PL, De Camilli P, Banker GA (1991) The distribution of synapsin I and synaptophysin in hippocampal neurons developing in culture. J Neurosci 11:1617-1626.

Fletcher TL, De Camilli P, Banker GA (1994) Synaptogenesis in hippocampal cultures: evidence indicating that axons and dendrites become competent to form synapses at different stages of neuronal development. J Neurosci 14:6695-6706.

Furshpan EJ, Mac Leish PR, O’Lague PH, Potter DD (1976) Chemical transmission between rat sympathetic neurons and cardiac myocytes developing in microcultures: evidence for cholinergic, adrenergic, and dual-function neurons. Proc Natl Acad Sci USA 73:4225-4229.

Futerman AH, Khanin R, Segel LA (1993) Lipid diffusion in neurons. Nature 362:119.

Galli T, Garcia EP, Mundigl O, Chilcote TJ, De Camilli P (1995) vand t-SNAREs in neuronal exocytosis: a need for additional components to define sites of release. Neuropharmacology 34:1351-1360.

Haydon PG, Zoran MJ (1994) Retrograde regulation of presynaptic development during synaptogenesis. J Neurobiol 25:694-706.

Herreros J, Miralles FX, Solsona C, Bizzini B, Blasi J, Marsal J (1995) Tetanus toxin inhibits spontaneous quantal release and cleaves VAMP/synaptobrevin. Brain Res 699:165-170.

Hua S-Y, Raciborska DA, Trimble WS, Charlton MP (1998) Different VAMP/synaptobrevin complexes for spontaneous and evoked transmitter release at the crayfish neuromuscular junction. J Neurophysiol 6:3233-3246.

Kraszewski K, Mundigl O, Daniell L, Verderio C, Matteoli M, De Camilli P (1995) Synaptic vesicle dynamics in living cultured hippocampal neurons visualized with CY3-conjugated antibodies directed against the lumenal domain of synaptotagmin. J Neurosci $15: 4328-4342$.

Laemmli UK (1970) Cleavage of structural proteins during the assembly of the head of bacteriophage T4. Nature 227:680-685.

Lledo P-M, Zhang X, Sudhof T, Malenka CR, Nicoll RA (1998) Postsynaptic membrane fusion and long-term potentiation. Science 279:399-403.

Maletic-Savatic M, Malinow R (1998) Calcium-evoked dendritic exocytosis in cultured hippocampal neurons. I. Trans-golgi networkderived organells undergo regulated exocytosis. J Neurosci 18:6803-6813.

Maletic-Savatic M, KoothanT, Malinow R (1998) Calcium-evocked dendritic exocytosis in cultured hippocampal neurons. II. Mediation by calcium/calmodulin-dependent protein kinase II. J Neurosci 18:6814-6821.

Matteoli M, Takei K, Cameron P, Johnston PA, Hurlbut P, Jahn R, Sudhof TC, De Camilli P (1991) Association of rab3 with synaptic vesicles at late stages of the secretory pathway. J Cell Biol 115:625-633.
Matteoli M, Takei K, Perin MS, Sudhof TC, De Camilli P (1992) Exo-endocytotic recycling of synaptic vesicles in developing processes of cultured hippocampal neurons. J Cell Biol 117:849-861.

Matteoli M, Verderio C, Rossetto O, Iezzi N, Coco S, Schiavo G, Montecucco C (1996) Synaptic vesicle endocytosis mediates the entry of tetanus neurotoxin into hippocampal neurons. Proc Natl Acad Sci USA 93:13310-13315.

Mellanby J, Thompson PA (1972) The effect of tetanus toxin at the neuromuscular junction in the goldfish. J Physiol (Lond) 224:407419.

Nichols BJ, Ungermann C, Pelham HR, Wickner WT, Haas A (1997) Homotypic vacuolar fusion mediated by $\mathrm{t}-$ and $\mathrm{v}$-SNAREs. Nature 387:199-202.

Nishimune A, Isaac JTR, Molnar E, Noel J, Nash SR, Tagaya N, Collingridge GL, Nakanishi S, Henley JM (1998) NSF binding to GluR2 regulates synaptic transmission. Neuron 21:87-97.

Osen Sand A, Staple JK, Naldi E, Schiavo G, Rossetto O, Petitpierre S, Malgaroli A, Montecucco C, Catsicas S (1996) Common and distinct fusion proteins in axonal growth and transmitter release. J Comp Neurol 367:222-234.

Osten P, Srivastava S, Inman GJ, Vilim FS, Khatri LM, Lee LM, States BA, Einheber S, Milner TA, Hanson PI, Ziff EB (1998) The AMPA receptor GluR2 C terminus can mediate a reversible, ATPdependent interaction with NSF and $\alpha$ - and $\beta$-SNAPs. Neuron 21:99-110.

Papini E, Rossetto O, Cutler DF (1995) Vesicle associated membrane protein (VAMP)/synaptobrevin 2 is associated with dense core secretory granules in PC12 neuroendocrine cells. J Biol Chem 270:1332-1336.

Petersen SA, Fetter RD, Noordermeer JN, Goodman CS, DiAntonio A (1997) Genetic analysis of glutamate receptors in Drosophila reveals a retrograde signal regulating presynaptic transmitter release. Neuron 19:1237-1248.

Pfenninger KH, Maylie-Pfenninger MF (1981) Lectin labeling of sprouting neurons. II. Relative movement appearance of glycoconjugates during plasmalemmal expansion. J Cell Biol 89:547-559.

Rossetto O, Gorza L, Schiavo G, Schiavo N, Scheller RH, Montecucco C (1996) VAMP/synaptobrevin isoforms 1 and 2 are widely and differentially expressed in nonneuronal tissues. J Cell Biol 132:167-179.

Schiavo G, Benfenati F, Poulain B, Rossetto O, Polverino de Laureto P, DasGupta BR, Montecucco C (1992) Tetanus and botulinum-B neurotoxins block neurotransmitter release by proteolytic cleavage of synaptobrevin. Nature 359:832-835.

Schiavo G, Rossetto O, Catsicas S, Polverino de Laureto P, DasGupta BR, Benfenati F, Montecucco C (1993a) Identification of the nerve terminal targets of botulinum neurotoxin serotypes A, D, and E. J Biol Chem 268:23784-23787.

Schiavo G, Shone CC, Rossetto O, Alexander FC, Montecucco C (1993b) Botulinum neurotoxin serotype $\mathrm{F}$ is a zinc endopeptidase specific for VAMP/synaptobrevin. J Biol Chem 268:11516-11519.

Schiavo G, Shone CC, Bennett MK, Scheller RH, Montecucco C (1995) Botulinum neurotoxin type C cleaves a single Lys-Ala bond within the carboxyl-terminal region of syntaxins. J Biol Chem 270:10566-10570.

Scholz KP, Miller RJ (1995) Developmental changes in presynaptic calcium channels coupled to glutamate release in cultured rat hippocampal neurons. J Neurosci 15:4612-4617.

Schulze KL, Broadie K, Perin MS, Bellen HJ (1995) Genetic and electrophysiological studies of syntaxin 1A demonstrate its role in nonneuronal secretion and neurotransmission. Cell 80:311-320.

Sollner T, Whiteheart SW, Brunner M, Erdjument-Bromage H, Geromanos S, Tempst P, Rothman JE (1993) SNAP receptors implicated in vesicle targeting and fusion. Nature 362:318-324.

Song I, Kamboj S, Xia J, Dong H, Liao D, Huganir RL (1998) Interaction of the $N$-ethylmaleimide-sensitive factor with AMPA receptors. Neuron 21:393-400.

Südhof TC (1995) The synaptic vesicle cycle: a cascade of proteinprotein interactions. Nature 375:645-653.

Sun YA, Poo MM (1987) Evoked release of acetylcoline from the growing embryonic neuron. Proc Natl Acad Sci USA 84:2540-2544.

Sweeney ST, Broadie K, Keane J, Niemann H, O'Kane CJ (1995) Targeted expression of tetanus toxin light chain in Drosophila specifically eliminates synaptic transmission and causes behavioral defects. Neuron 14:341-351. 
Towbin H, Staehelin T, Gordon J (1979) Electrophoretic transfer of proteins from polyacrylamide gels to nitrocellulose sheets: procedures and some applications. Proc Natl Acad Sci USA 76:4350-4354.

Verderio C, Coco S, Fumagalli G, Matteoli M (1995) Calciumdependent glutamate release during neuronal development and synaptogenesis: different involvement of $\omega$-Aga-IVA and $\omega$-C tx-GVIA sensitive channels. Proc Natl Acad Sci USA 92:6449-6453.

Verderio C, Coco S, Rossetto O, Montecucco C, Matteoli M (1999a) Internalization and proteolytic action of Botulinum toxins in CNS neurons and astrocytes. J Neurochem, in press.

Verderio C, Coco S, Pravettoni E, Bacci A, Matteoli M (1999b) Synaptogenesis in hippocampal cultures. Cell Mol Life Sci, in press.
Walch-Solimena C, Blasi J, Edelmann L, Chapman ER, von Mollard GF, Jahn R (1995) The t-SNARE syntaxin 1 and SNAP-25 are present on organelles that participate in synaptic vesicle recycling. J Cell Biol 128:637-645.

Young SH, Poo MM (1983) Spontaneous release of transmitter from growth cones of embryonic neurones. Nature 368:140-144.

Zakharenko S, Popov S (1998) Dynamics of axonal microtubules regulate the topology of new membrane insertion into the growing neurites. J Cell Biol 143:1077-1086.

Zakharenko S, Chang S, O'Donoghue M, Popov SV (1999) Neurotransmitter secretion along growing nerve processes: comparison with synaptic vesicle exocytosis. J Cell Biol 114:507-518. 